\title{
Involvement of ribonucleotide reductase-M1 in 5-fluorouracil-induced DNA damage in esophageal cancer cell lines
}

\author{
YOSHIRO AOKI $^{1}$, KENJI SAKOGAWA $^{1}$, JUN HIHARA $^{1}$, MANABU EMI $^{1}$, YOICHI HAMAI ${ }^{1}$, \\ KAZUTERU KONO ${ }^{2}$, LIN SHI ${ }^{2}$, JIYING SUN ${ }^{2}$, HIROYUKI KITAO ${ }^{3}$, TSUYOSHI IKURA ${ }^{4}$, \\ HIROYUKI NIIDA $^{5}$, MAKOTO NAKANISHI ${ }^{6}$, MORIHITO OKADA $^{1}$ and SATOSHI TASHIRO ${ }^{2}$
}

\begin{abstract}
Departments of ${ }^{1}$ Surgical Oncology and ${ }^{2}$ Cellular Biology, Research Institute for Radiation Biology and Medicine, Hiroshima University, Minami-ku, Hiroshima; ${ }^{3}$ Department of Molecular Oncology, Graduate School of Medical Sciences, Kyushu University, Higashi-ku, Fukuoka; ${ }^{4}$ Department of Mutagenesis, Radiation Biology Center, Kyoto University, Sakyo-ku, Kyoto; ${ }^{5}$ Department of Molecular Biology, Hamamatsu University School of Medicine, Higashi-ku, Hamamatsu; ${ }^{6}$ Department of Experimental Pathology and Tumor Biology, Graduate School of Medical Sciences, Nagoya City University, Mizuho-ku, Nagoya, Japan
\end{abstract}

Received January 11, 2013; Accepted February 22, 2013

DOI: 10.3892/ijo.2013.1899

\begin{abstract}
Fluorouracil (5-FU) is one of the most well established chemotherapeutic agents in the treatment of esophageal cancer. Ribonucleotide reductase M1 (RRM-1) is the rate-limiting enzyme in de novo DNA synthesis, and has been considered to play an important role in the 5-FU metabolic pathway. However, the means by which RRM-1 participates in the anticancer effects of 5-FU and cisplatin (CDDP) have not been well studied. Here, we show that RRM-1 significantly contributes to the induction of DNA damage by 5-FU in esophageal cancer cell lines. An assay of $\gamma-\mathrm{H} 2 \mathrm{AX}$ focus formation, a marker of DNA damage, after 5-FU treatment revealed good correlation with the levels of RRM-1 protein expression. Moreover, the increased sensitivity and RAD51 focus formation induced by the combination treatment of 5-FU and CDDP were significantly repressed by RRM-1 depletion. These results suggest that RRM-1 is involved not only in the induction of DNA damage by 5-FU but also in the synergistic cytotoxic effect in the combination therapy of 5-FU and CDDP.
\end{abstract}

\section{Introduction}

Esophageal cancer is one of the most aggressive types of cancer and the eighth most common cause of cancer-related death in

Correspondence to: Professor Satoshi Tashiro, Department of Cellular Biology, Research Institute for Radiation Biology and Medicine, Hiroshima University, 1-2-3 Kasumi, Minami-ku, Hiroshima 734-8551, Japan

E-mail: ktashiro@hiroshima-u.ac.jp

Key words: ribonucleotide reductase, cisplatin, 5-fluorouracil, $\gamma$-H2AX, RAD51 the world (1,2). In spite of recent advances in the development of anticancer and molecular target drugs, the combination regimen of 5-fluorouracil (5-FU) and cisplatin (CDDP) still plays an important role in the treatment of esophageal cancer, as the gold standard chemotherapy.

The nucleoside analogue 5-FU is converted to several active metabolites, including fluorouridine triphosphate (FUTP), fluorodeoxyuridine triphosphate (FdUTP) and fluorodeoxyuridine monophosphate (FdUMP), to exert its cytotoxic activity (Fig. 1). FUTP is largely incorporated into the RNA strand, preventing the processing and function of various types of RNA including mRNA, rRNA, tRNA and snRNA to exert anticancer effect of 5-FU (3). Recently, several lines of evidence suggest that these metabolites of 5-FU also interrupt DNA metabolism by misincorporation or enzyme inhibition (3). For example, FdUMP disturbs DNA replication by inhibiting the activity of thymidylate synthetase (TS), through the depletion of dTMP. 5-FU may also generate DNA damage, such as DNA single-strand breaks and/or DNA double strand breaks (DSBs) via the collapse of stalled replication forks. Although the induction of DNA damage has been shown to play a role in the anticancer effect of 5-FU (4-6), the means by which the metabolites of 5-FU exert their anticancer effects through the disturbance of DNA or RNA metabolism remain obscure (7).

Several 5-FU related enzymes, such as dihydropyrimidine dehydrogenase (DPD), orotate phosphoribosyl transferase (OPRT), thymidine phosphorylase (TP) and ribonucleotide reductase (RNR), are considered as major determinants of the outcome of 5-FU treatment (8-10) (Fig. 1). Among these enzymes, ribonucleotide reductase, composed of large subunit RRM-1 and small subunit RRM-2, is the rate-limiting enzyme in de novo DNA synthesis (11). RNR converts fluorouridine diphosphate (FUDP) to fluorodeoxyuridine diphosphate (FdUDP), which preferentially affects DNA metabolism. Recently, RNR has also been shown to play an important role 


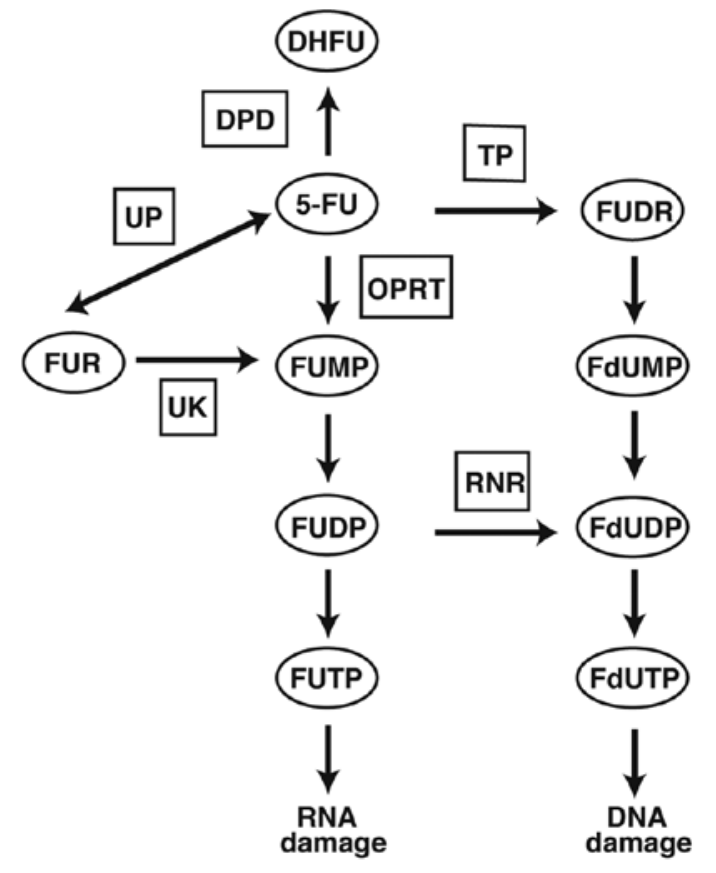

Figure 1. Metabolism of 5-FU.

in DNA repair (12). However, how RNR is involved in the regulation of the anticancer effect of 5-FU remains to be clarified.

DNA damage, such as stalled replication forks and DSBs, induces the phosphorylation of histone H2AX on Ser-139 $(\gamma-\mathrm{H} 2 \mathrm{AX})$, and the focus formation of $\gamma$-H2AX $(\gamma-\mathrm{H} 2 \mathrm{AX}$ foci) (13). $\gamma-\mathrm{H} 2 \mathrm{AX}$ is required to retain DNA repair proteins and checkpoint proteins at damaged sites, for the efficient processing of damaged DNA $(14,15)$. Homologous recombination (HR) is a versatile DNA repair mechanism, because it can promote the elimination of a variety of lesions, including DSBs, single-strand gaps and stalled DNA replication forks $(16,17)$. RAD51 is one of the key proteins for DNA repair by HR, since it mediates homologous pairing and strand exchange between DNA duplexes (18). RAD51 has also been shown to form nuclear foci at DSB sites (19). Since many anticancer drugs induce DNA damage, analyses of DNA repair proteins, such as $\gamma$-H2AX and RAD51, could be useful to predict the outcome of anticancer drugs (20-22). Recently, a dose-dependent increase in the number of $\gamma$-H2AX foci after 5-FU treatment was reported (23). CDDP also induces the $\gamma-\mathrm{H} 2 \mathrm{AX}$ and RAD51 foci by the formation of interstrand and intrastrand DNA crosslinks, which lead to the formation of DSBs when they meet replication forks (24-26). Although the combination regimen of 5-FU and CDDP shows synergistic effects both in vitro and in vivo, the involvement of DNA damage in this effect remained to be clarified $(27,28)$.

In this study, we investigated the mechanism of the anticancer effect of 5-FU, by analyzing the kinetics of $\gamma-\mathrm{H} 2 \mathrm{AX}$ and RAD51 foci formation in esophageal cancer cells. RRM-1 was required for the $\gamma-\mathrm{H} 2 \mathrm{AX}$ focus formation after 5-FU treatment. Moreover, RRM-1 enhanced the formation of RAD51 foci, but not $\gamma$-H2AX foci, when cells were treated with 5-FU and CDDP in combination. These findings clearly suggest that RRM-1 is involved in the anticancer effect of 5-FU by modulating DNA repair in human esophageal cancer cells.

\section{Materials and methods}

Cell lines, culture medium and chemicals. Two esophageal carcinoma cell lines (TE1 and TE11) were obtained from the Cell Resource Center for the Biomedical Research Institute of Development, Aging and Cancer (Tohoku University, Sendai, Japan). TE1 is a well differentiated squamous cell carcinoma, and TE11 is a moderately differentiated squamous cell carcinoma. These cell lines were routinely grown in RPMI-1640 (Invitrogen, Carlsbad, CA, USA) supplemented with $10 \%$ fetal bovine serum, and incubated at $37^{\circ} \mathrm{C}$ in a humidified atmosphere of $5 \% \mathrm{CO}_{2}$ in air. 5-FU (Kyowa Hakko Co., Japan) and CDDP (Nihonkayaku Co., Japan) were dissolved in PBS at $1 \mathrm{mM}$.

Clonogenic assay for cell survival. The drug sensitivity of the cells was assessed by their colony forming ability. In brief, approximately $2.0 \times 10^{3}$ cells were seeded in a $60-\mathrm{mm}$ tissue culture dish and incubated overnight. To assess the synergistic effect of 5-FU and CDDP, the concomitant treatment regimen involved the simultaneous treatment of cells with 5-FU (1-3 $\mu \mathrm{M})$ for $24 \mathrm{~h}$ and CDDP $(1-5 \mu \mathrm{M})$ for $1 \mathrm{~h}$, alongside suitable controls of cells treated with appropriate doses of CDDP $(0-10 \mu \mathrm{M})$ alone for $1 \mathrm{~h}$, or $5-\mathrm{FU}(0-10 \mu \mathrm{M})$ alone for $24 \mathrm{~h}$. The cells were washed in PBS and cultured for 7-10 days in a 5\% $\mathrm{CO}_{2}$ atmosphere at $37^{\circ} \mathrm{C}$ in air. The colonies were fixed with $100 \%$ methanol for $10 \mathrm{~min}$, and stained with Giemsa in phosphate buffer ( $\mathrm{pH}$ 6.4). Colonies composed of 50 or more cells were scored as survivors, and the surviving fraction for a given treatment dose was calculated as the relative plating efficiency of treated versus untreated (control) cultures. All experiments were performed three times and yielded similar results.

Dose-response curves were analyzed using the CalcuSyn Software (Biosoft), which is based on the median effect model of Chou and Talalay. A combination index (CI) of 1 indicated an additive drug interaction, whereas a $\mathrm{CI}$ of $>1$ was antagonistic and a score lower than 1 was synergistic.

Immunofluorescence analysis. The cells were seeded in 6-well plates containing coverslips, and were treated with $3 \mu \mathrm{M} 5-\mathrm{FU}$ for $24 \mathrm{~h}, 5 \mu \mathrm{M}$ CDDP for $1 \mathrm{~h}$, or the concomitant treatment, and were allowed to recover for the indicated periods. The coverslips were then fixed with $4 \%$ paraformadehyde at room temperature for $10 \mathrm{~min}$. The cells were washed twice with $0.1 \%$ Triton X-100 in PBS (PBS-T), permeablized with $0.5 \%$ Triton X-100 and $0.1 \%$ SDS in PBS for $10 \mathrm{~min}$, and washed with $0.05 \%$ glycine and $0.1 \%$ BSA in PBS (PBS+). The coverslips were incubated with the primary antibody at $37^{\circ} \mathrm{C}$ for $90 \mathrm{~min}$. The primary antibodies were used at the following ratios: anti-RAD51 (Calbiochem, La Jolla, CA, USA), 1:500; anti- $\gamma$-H2AX (Upstate Biotechnology, Lake Plasid, NY, USA) 1:40,000. The coverslips were incubated at $37^{\circ} \mathrm{C}$ for $1 \mathrm{~h}$ with the secondary antibody, either sheep-antimouse-Cy3 (BioSource, USA) at 1:400 or goat-anti-rabbit-FITC (Jackson ImmunoResearch Laboratories Inc., West Grove, PA, USA) at 1:500. DNA counter staining was accomplished with DAPI (4', 6-diamidino-2-phenylindole). After staining, the coverslips were sealed with VectaShield (Vector Laboratories Inc., Burlingame, CA, USA) and stored in the dark.

Images were obtained with a Zeiss Axiovert $200 \mathrm{M}$ fluorescence microscope, using the AxioVision Rel 4.4 software (Carl Zeiss, Germany). Cells were scored as positive for damage- 
induced foci if there were $\geq 15$ spots per cell for $\gamma-\mathrm{H} 2 \mathrm{AX}$ and $\geq 5$ for RAD51. About 200 cells were observed for each regimen, and the averaged data are from three independent experiments.

siRNA treatment. TE11 cells were transfected with either the non-targeting control (NT) siRNA (Dharmacon, Inc., USA) or the siRNA for human RRM-1 (GGAUCGCUGUCUCUAA CUUtt). The siRNA for human RRM-1 was kindly provided by Dr H. Niida (Department of Molecular Biology, Hamamatsu University School of Medicine, Shizuoka, Japan) (12). Transfection was performed $24 \mathrm{~h}$ before 5-FU and/or CDDP treatment using Lipofectamine 2000 (Invitrogen) according to the manufacturer's instructions, and the final concentrations of both the NT siRNA and RRM-1 siRNA were $1 \mathrm{nM}$ in OptiMEM (Invitrogen) medium per well.

Immunoblot analysis. Cells were harvested and lysed in Laemmli's buffer. Total cell protein extracts were fractionated by SDS-PAGE using a multigel II (Cosmo Bio, Japan), and were electrophoretically transferred onto PVDF membranes. The membranes were blocked with $5 \%$ non-fat dried milk in TBS containing $0.1 \%$ Tween-20 (TBS-T) for $1 \mathrm{~h}$. The membranes were then incubated with primary antibodies against $\beta$-actin (Sigma, St. Louis, MO, USA), RRM-1 or RRM-2 (Santa Cruz Biotechnology Inc., Santa Cruz, CA, USA) for $1 \mathrm{~h}$. The membranes were then washed three times with TBS-T and incubated with the appropriate secondary antibodies. After three washes with TBS-T, the immunoreactive proteins were visualized by enhanced chemiluminescence (Amersham Biosciences, Uppsala, Sweden).

Flow cytometry for $\gamma-H 2 A X$ analysis. The cells were harvested with $2 \%$ trypsin and washed three times in cold PBS. After permeation, the cells were incubated with the anti- $\gamma$-H2AX antibody (1: 40,000 dilution) for $1 \mathrm{~h}$. The cells were washed and resuspended in the secondary antibody, sheep anti-mouse-Cy3 (1:400 dilution), for $30 \mathrm{~min}$. After fixation in $0.5 \%$ paraformadehyde, the fluorescence emitted by $\mathrm{Cy} 3$ was measured by flow cytometry.

Statistical analysis. The differences in the cell viability and the percentage of focus formation of $\gamma-\mathrm{H} 2 \mathrm{AX}$ and RAD51 between samples were evaluated with the Student's unpaired t-test. $\mathrm{P}<0.05$ was regarded as statistically significant.

\section{Results}

Induction of DNA damage with 5-FU in esophageal cancer cell lines. To study the mechanisms of the anticancer activity of 5-FU, we investigated the survival of two esophageal cell lines, TE1 and TE11, after 5-FU treatment by a clonogenic assay. Representative dose-survival responses of 5-FU are depicted in Fig. 2A. The Dm values for 5-FU were estimated as 3.6 and $2.7 \mu \mathrm{M}$ in TE11 and TE1, respectively. This indicates that there are no significant differences in the 5-FU sensitivities between these two cell lines.

Next, we examined the role of the induction of DNA damage in the anticancer effect of 5-FU $(4,5)$. The formation of $\gamma$-H2AX foci at damaged sites is one of the highly sensitive markers of DNA damage induced by ionizing radiation and/or chemo- therapeutic agents, such as CDDP (21). Therefore, we performed immunofluorescence staining of 5-FU-treated TE11 cells, using anti- $\gamma$-H2AX antibodies (Fig. 2B). The percentage of cells with $\gamma-\mathrm{H} 2 \mathrm{AX}$ foci increased in a time-dependent manner. The peak was observed at $24 \mathrm{~h}$ after 5-FU exposure in TE11 cells (Fig. 2C). The flow cytometry analysis of $\gamma$-H2AX confirmed the increase in $\gamma$-H2AX positive TE11 cells after 5-FU treatment (Fig. 2D). In contrast to TE11, TE1 cells failed to show an increase in $\gamma$-H2AX foci positive cells after 5-FU treatment (Fig. 2B-D). These findings suggest that 5-FU induces more DNA damage in TE11 cells, as compared to TE1 cells. Since the sensitivity of TE11 cells to 5-FU is similar to that of TE1 (Fig. 2A), the mechanism by which 5FU exerts its anticancer effect could be different in these esophageal cell lines.

Metabolism of 5-FU in the two esophageal cancer cell lines. The difference in the induction of DNA damage by 5-FU in TE1 and TE11 cells led us to examine the enzymes involved in the metabolism of the drug (3). The pharmacogenetic variability of 5-FU-related enzymes, such as DPD, OPRT, TS and RNR, is associated with the anticancer activity of 5-FU (8-10). Among these enzymes, RNR is required to supply ribonucleotides for both DNA replication and repair (12). Thus, we examined the protein levels of the RNR subunits, RRM-1 and RRM-2, in the two cell lines, TE1 and TE11. The immunoblot analyses using the anti-RRM-1 antibody revealed that TE11 expressed a significantly higher level of RRM-1, as compared to TE1 cells. The expression of RRM-2 was slightly higher in TE11 cells than TE1 cells (Fig. 3A). The treatment of cells with 5-FU did not alter the expression of these enzymes. These results suggest that the high expression of RNR, especially RRM-1, in TE11 facilitates the induction of DNA damage, by converting 5-FU to 5-FdU.

To confirm the involvement of RRM-1 in the induction of DNA damage by 5-FU, we next examined the effect of RRM-1 depletion in TE11 cells. RRM-1 depletion by the siRNA was confirmed by an immunoblot analysis of TE11 cells (Fig. 3B). To investigate the effect of RRM-1 depletion on DNA damage in TE11 cells, we first performed immunofluorescence staining of $\gamma$-H2AX after 5-FU treatment (Fig. 3C). The RRM-1 depletion by siRNA significantly repressed the $\gamma-\mathrm{H} 2 \mathrm{AX}$ focus formation after 5-FU treatment ( $\mathrm{p}<0.01)$ (Fig. 3D). This finding strongly supports the notion that RRM-1 is involved in the induction of DNA damage in TE11 cells after 5-FU treatment.

Next we investigated the survival of these cells after 5-FU treatment by a clonogenic assay, to determine whether RRM-1 depletion is involved in 5-FU sensitivity. Representative dosesurvival responses of 5-FU are depicted in Fig. 3E. The Dm values for 5-FU were estimated as $3.34 \mu \mathrm{M}$ in RRM-1 siRNA treated cells and $3.44 \mu \mathrm{M}$ in control cells. Therefore, a significant change in 5-FU sensitivity by the depletion of RRM-1 was not observed. The reduced anticancer activity of 5-FU, through the lower induction of DNA damage, might be compensated by the increased disturbance of RNA metabolism by the depletion of RRM-1.

Induction of DNA damage and damage response with the combination of 5-FU and CDDP in esophageal cancer cell lines. Having established that 5-FU induces DNA damage in TE11 cells, but not in TE1 cells, we elucidated the mechanism of the synergistic effect of the combination regimen of 5-FU and 
A

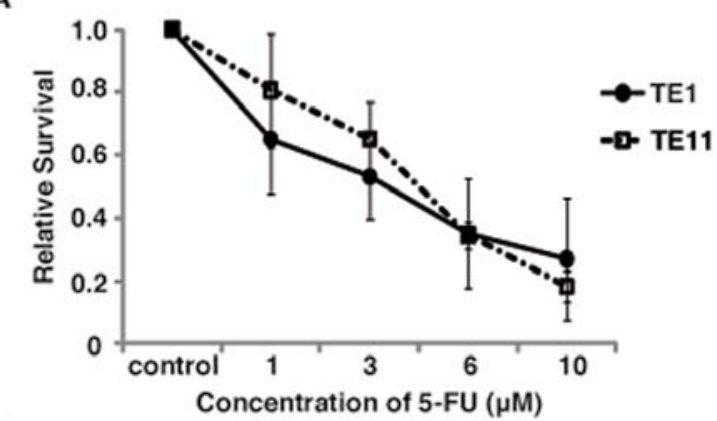

B
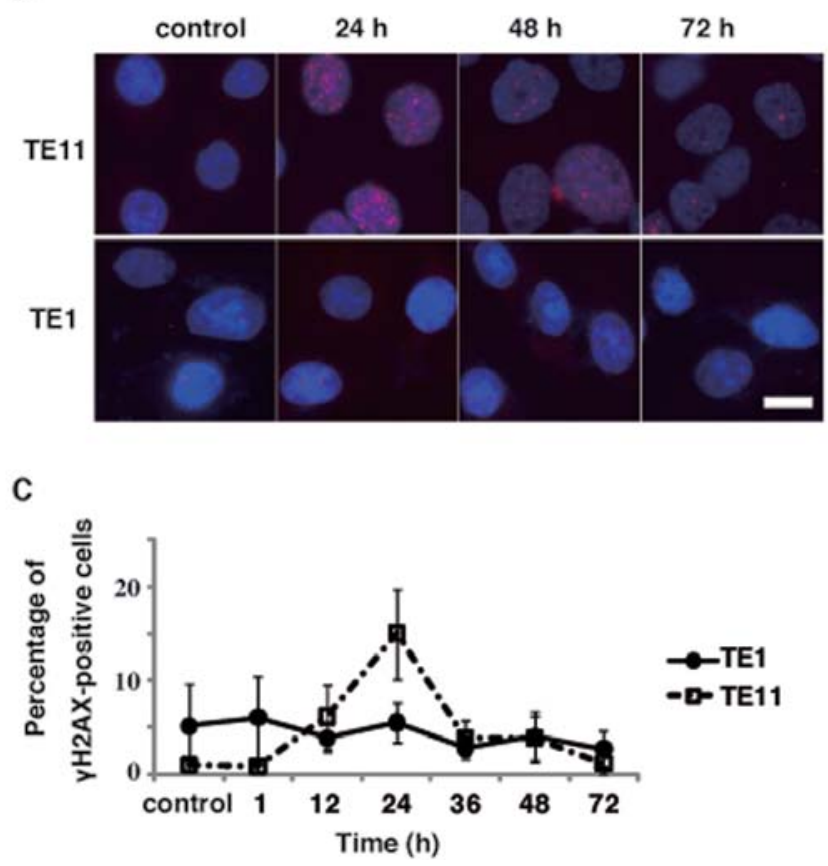

D
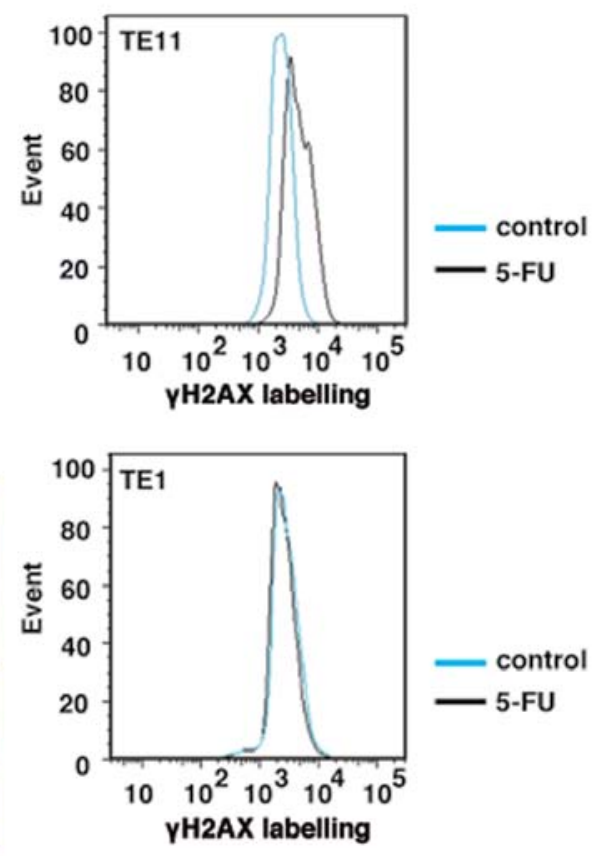

Figure 2. Toxicity in relation to the kinetics of $\gamma$-H2AX and RAD51 foci formation in esophageal cancer cell lines treated with 5-FU. (A) Cytotoxic effects on the TE11 and TE1 cell lines after a 24-h 5-FU exposure were determined using the clonogenic assay. Results are the means of at least three independent experiments, and error bars show the standard deviation of the mean. (B) Time-course of antibody-stained images of TE11 and TE1 cell lines treated with $3 \mu \mathrm{M} 5$-FU for $24 \mathrm{~h}$. $\gamma$-H2AX and DNA (DAPI) are shown in red and blue, respectively. Scale bars, $20 \mu \mathrm{m}$. (C) Kinetics of $\gamma$-H2AX foci formation in TE11 and TE1 cell lines after a 24-h exposure to $3 \mu \mathrm{M}$ 5-FU. All results are the means of three independent experiments, and error bars show the standard deviation of the mean. (D) Flow cytometric detection of $\gamma-\mathrm{H} 2 \mathrm{AX}$ in TE11 and TE1 cell lines treated with 5-FU for $24 \mathrm{~h}$.

CDDP. First we performed clonogenic assays to determine if the synergistic effect could be observed in the TE11 cell line. As a result, the co-incubation of TE11 cells with $5 \mu \mathrm{M}$ CDDP and $3 \mu \mathrm{M}$ 5-FU showed the most significant synergistic inhibition of cell growth (Fig. 4A and B).

Since CDDP is a widely used platinum-containing anticancer drug with an activity mechanism that involves the formation of DNA damage, we next examined the induction of DNA damage in TE11 cells after treatments with either CDDP alone or $3 \mu \mathrm{M} 5-\mathrm{FU}$ and $5 \mu \mathrm{M}$ CDDP. Immunofluorescence staining of TE11 cells with the anti- $\gamma$-H2AX antibody revealed that CDDP alone and in the combination treatment induced $\gamma$-H2AX focus formation in a time-dependent manner (Fig. 5A and B). The percentage of $\gamma$-H2AX foci was slightly higher in the cells treated with the combination of 5-FU and CDDP than in those treated with CDDP or 5-FU alone. The peak was observed at $24 \mathrm{~h}$ after the combination regimen, and was similar to 5-FU alone (Fig. 5B). In contrast, the peak of $\gamma-\mathrm{H} 2 \mathrm{AX}$ focus formation was observed at $12 \mathrm{~h}$ after CDDP treatment. Moreover, the increase in the percentage of $\gamma-\mathrm{H} 2 \mathrm{AX}$ focus positive cells by the combinational treatment was additive. Therefore, the synergic effect may be due to a modulation of DNA repair, rather than an increase in the induction of DNA damage by the combinational treatment.

To further investigate the involvement of DNA damage induced by the combinational treatment in the synergic effect, we examined the kinetics of RAD51 focus formation, another DNA damage marker formed by a recombinational repair protein (Fig. 5A). Immunofluorescence analyses using an anti-RAD51 antibody revealed that the percentage of RAD51 foci in TE11 cells was not significantly increased after a treatment with 5-FU alone. While CDDP treatment slightly increased the percentage of RAD51 foci positive cells, the combinational treatment with 5-FU and CDDP drastically induced the RAD51 focus formation in TE11 cells (Fig. 5C). Interestingly, the increased focus formation of RAD51 persisted longer than that of $\gamma-\mathrm{H} 2 \mathrm{AX}$ after the combinational treatment. Moreover, increase of the focus formation of RAD51 is more significant 
A

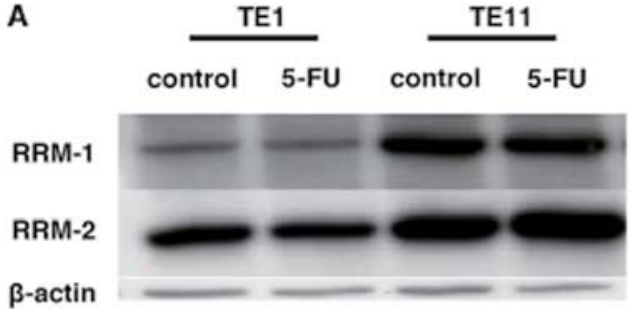

C

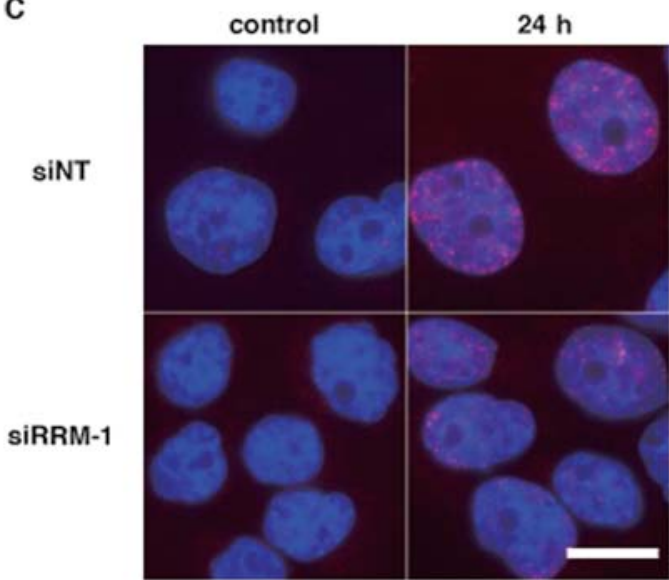

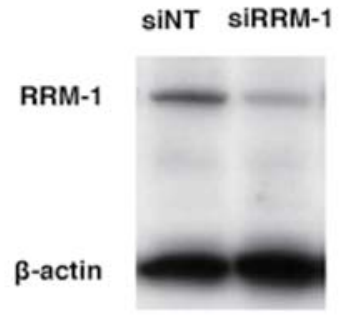

D



E

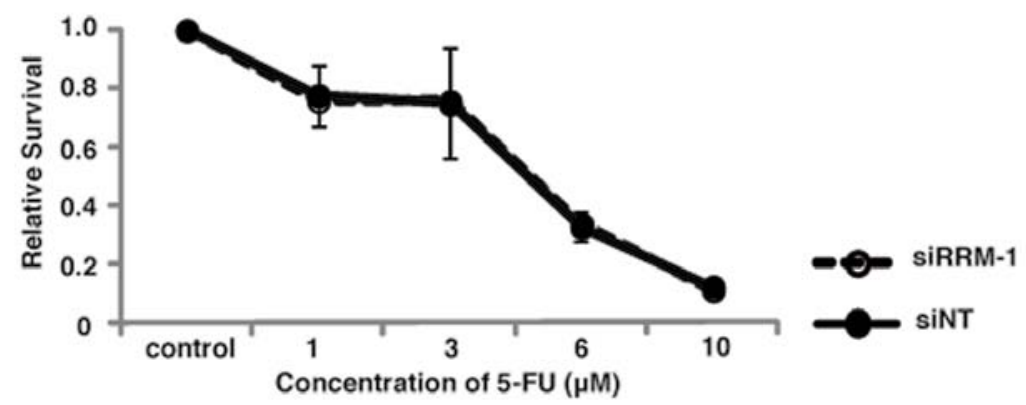

Figure 3. Expression levels of RNR subunits and effect of RRM-1 depletion in the TE11 cell line. (A) Comparison of the RRM-1 and RRM-2 protein expression levels between TE11 and TE1 cells treated or untreated with $3 \mu \mathrm{M}$ 5-FU for $24 \mathrm{~h}$. (B) Expression levels of RRM-1 in TE11 cells transfected with RRM-1 siRNA (siRRM-1) or non-target siRNA (siNT). (C) Immunofluorescence staining of TE11 cells, using anti- $\gamma$-H2AX antibodies. Cells were transfected with RRM-1 siRNA or NT siRNA before 5-FU treatment. $\gamma$-H2AX and DNA are shown in red and blue, respectively. Scale bars, $20 \mu \mathrm{m}$. (D) Percentage of $\gamma$-H2AX-positive cells, following a $3 \mu \mathrm{M}$ 5-FU treatment for $24 \mathrm{~h}$. All results are the means of three independent experiments, and error bars show the standard deviation of the mean. (E) Clonogenic assay of TE11 cells expressing either RRM-11 siRNA or NT siRNA, and treated with 5-FU for $24 \mathrm{~h}$. Results are the means of at least three independent experiments, and error bars show the standard deviation of the mean.

than that of $\gamma-\mathrm{H} 2 \mathrm{AX}$ by the combinational treatment compared to either CDDP or 5-FU alone (Fig. 5B and C). Therefore, the modulation of DNA repair, rather than the increased induction of DNA damage, could contribute more to the synergic effect of the combinational treatment observed in TE11 cells.

RRM-1 is involved in the synergic effect of the combination treatment. We next examined if RRM-1 is involved in the synergic effect of the combinational treatment, since RRM-1 plays a role in the 5-FU-induced DNA damage in TE11 cells (Fig. 3). We performed a clonogenic assay of RRM-1-depleted cells after the 5-FU/CDDP treatment. As expected, the depletion of RRM-1 with siRNA significantly increased the survival of
TE11 cells after the combinational treatment $(\mathrm{p}<0.05)$ (Fig. 6A). Since RRM-1 depletion did not affect the 5-FU or CDDP sensitivity of TE11 (Figs. 3E and 7C, p=0.90), this finding suggests the involvement of RRM-1 in the synergic effect of the combination regimen with 5-FU and CDDP in TE11 cells.

To study the role of RRM-1 in the synergic effect, we examined the effect of RRM-1 depletion on the induction of DNA damage after the combinational treatment of TE11 cells. Immunofluorescence staining with the anti- $\gamma$-H2AX antibody revealed that the depletion of RRM-1 by siRNA did not significantly change the percentage of TE11 cells with $\gamma$-H2AX foci after the combinational treatment ( $\mathrm{p}=0.86$, Fig. $6 \mathrm{~B}$ and $\mathrm{D})$. The $\gamma$-H2AX focus formation was not significantly increased by 
A

\begin{tabular}{l|c|c} 
& $5-\mathrm{FU} 1 \mu \mathrm{M}$ & $5-\mathrm{FU} 3 \mu \mathrm{M}$ \\
\hline $\operatorname{CDDP} 1 \mu \mathrm{M}$ & $0.6402 \pm 0.1984(1)$ & $0.5104 \pm 0.0435(2)$ \\
\hline $\operatorname{CDDP} 5 \mu \mathrm{M}$ & $0.5236 \pm 0.0959(3)$ & $0.3107 \pm 0.0293(4)$ \\
\hline
\end{tabular}

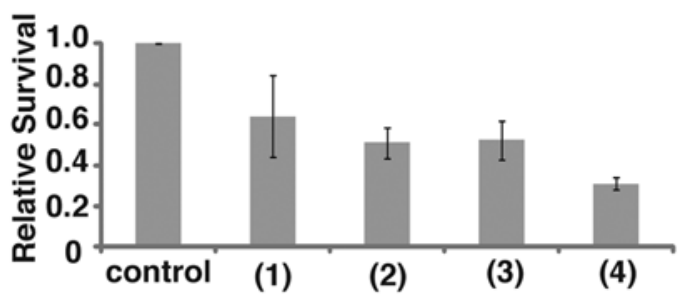

B

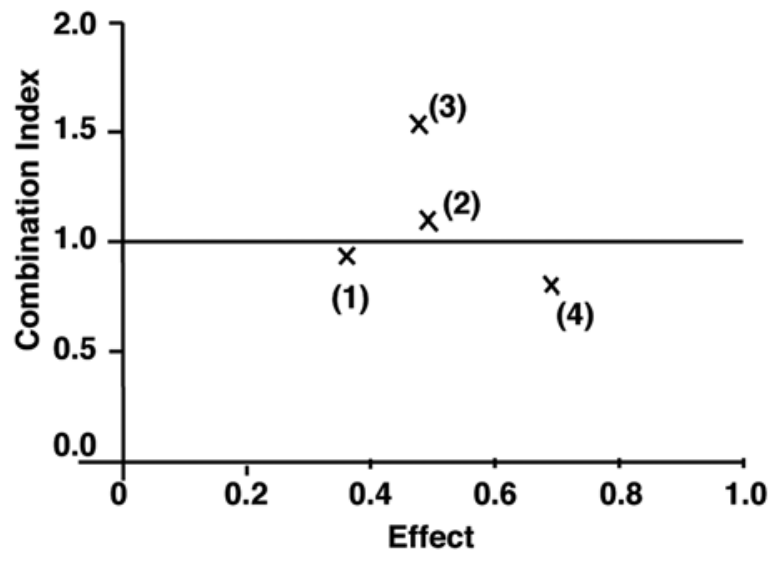

Figure 4. Toxicity in TE11 cell lines treated with the combination of 5-FU and CDDP. (A) Several combinations of drug concentrations were tested, and their effects are shown. (B) Combination index is shown. Particularly, co-incubation of TE11 cells with $5 \mu \mathrm{M}$ CDDP and $3 \mu \mathrm{M} 5$-FU showed the most synergistic inhibition of cell growth $(\mathrm{Cl}=0.788)$.

A
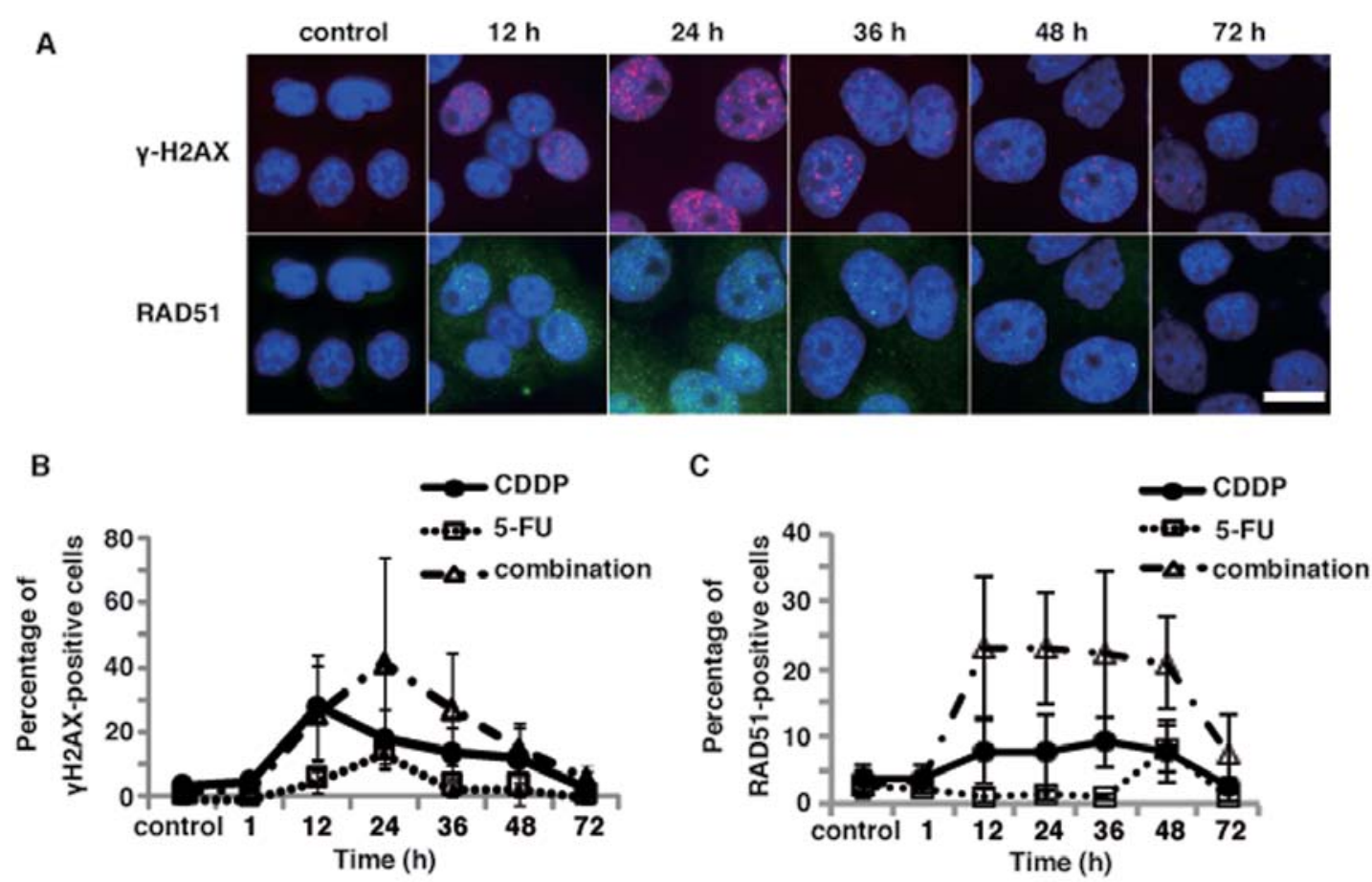

Figure 5. Toxicity in relation to the kinetics of $\gamma$-H2AX and RAD51 in TE11 cell lines treated with the combination of 5-FU and CDDP. (A) Immunostaining of TE11 cells using anti- $\gamma-\mathrm{H} 2 \mathrm{AX}$ and RAD51 antibodies. Cells were treated with $3 \mu \mathrm{M} 5$-FU for $24 \mathrm{~h}$ and $5 \mu \mathrm{M} \mathrm{CDDP}$ for $1 \mathrm{~h} . \gamma$-H2AX, RAD51 and DNA are shown in red, green and blue, respectively. Scale bars, $20 \mu \mathrm{m}$. (B and C) Kinetics of $\gamma$-H2AX and RAD51 focus formation in TE11 cells, after a 24-h exposure to $3 \mu \mathrm{M}$ 5-FU and a 1-h exposure to $5 \mu \mathrm{M}$ CDDP. All results are the means of three independent experiments, and error bars show the standard deviation of the mean.

the combinational treatment, as compared to the treatments with 5-FU or CDDP alone (Fig. 5B). Moreover, the $\gamma$-H2AX focus formation after CDDP treatment was not affected by the RRM-1 depletion (Fig. 7A, p=0.21). Taken together, these findings strongly suggest that the facilitation of DNA damage induction by RRM-1 is not involved in the synergic effect of the combinational treatment.

If the synergic effect of the combinational treatment was not due to increased DNA damage by the combinational treatment, then the modulation of the repair system could be involved in this effect. To test this hypothesis, we next examined the effect of the depletion of RRM-1 on the RAD51 focus formation (Fig. 6C). Immunfluorescence analyses using an anti-RAD51 antibody revealed that, in contrast to $\gamma-\mathrm{H} 2 \mathrm{AX}$, RAD51 focus formation was significantly repressed by the depletion of RRM-1 in TE11 cells, after the combinational treatment with 5-FU and CDDP $(\mathrm{p}<0.01)$ (Fig. 6E). In contrast, the RAD51 focus formation in TE11 cells treated with CDDP alone was not interrupted by the depletion of RRM-1 (Fig. 7B, p=0.15). Taken together, these findings suggest that RRM-1 is involved in the repair of the DNA 
A

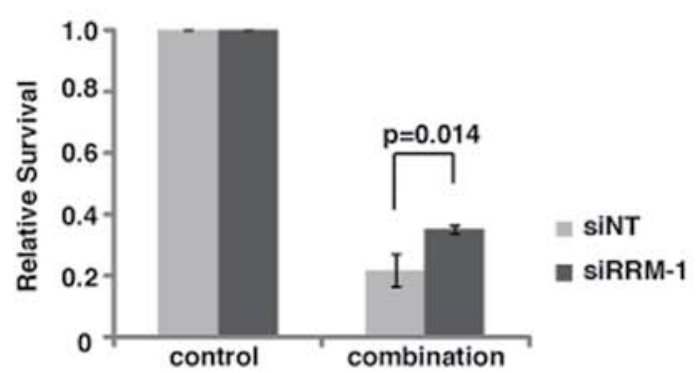

B

Y-H2AX

control

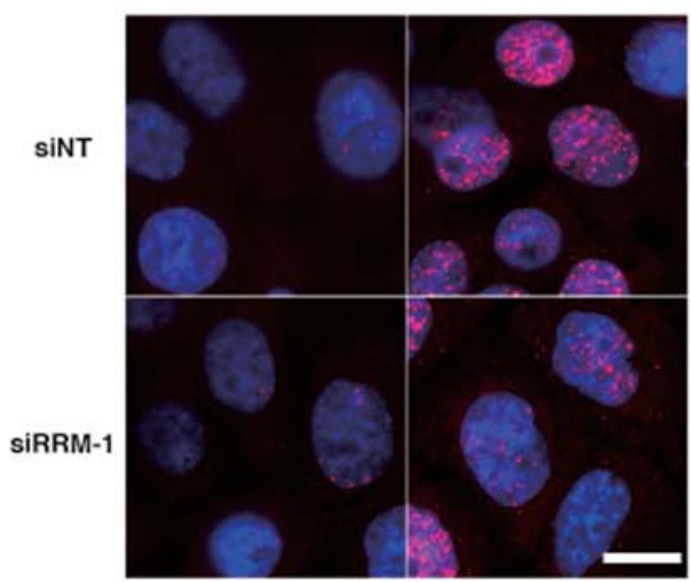

D

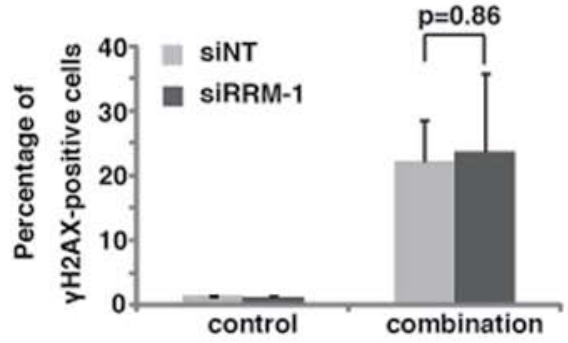

C RAD51

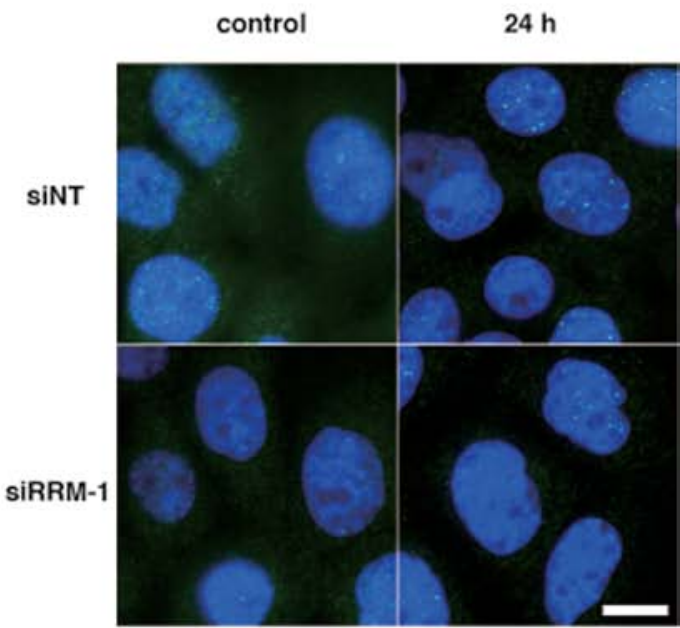

E

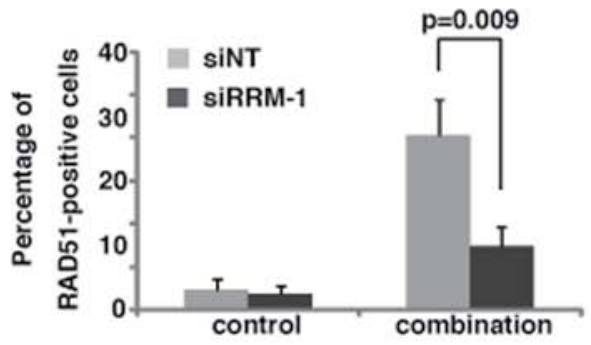

Figure 6. Effect of RRM-1 depletion on the induction of $\gamma$-H2AX and RAD51 focus formation in TE11 cells treated with the combination regimen. (A) The cytotoxicity of cells expressing either the NT siRNA (siNT) or RRM-1 siRNA (siRRM-1) was determined, using the clonogenic assay. Results are the means of at least three independent experiments, and error bars show the standard deviation of the mean. (B and C) Immunostaining of TE11 cells transfected with RRM-1 siRNA and NT siRNA, using anti- $\gamma$-H2AX and RAD51 antibodies. Cells were treated with $3 \mu \mathrm{M} 5$-FU for 24 h and $5 \mu$ M CDDP for 1 h. $\gamma$-H2AX, RAD51 and DNA are shown in red, green and blue, respectively. Scale bars, $20 \mu \mathrm{m}$. (D) Percentage of $\gamma$-H2AX foci-positive RRM-1 depleted cells, before and after the combinational treatment. All results are the means of three independent experiments, and error bars show the standard deviation of the mean. (E) Percentage of RAD51 foci-positive cells. All results are the means of three independent experiments, and error bars show the standard deviation of the mean.

damage induced by the combinational treatment, but not by the CDDP treatment alone. Therefore, the modulation of DNA repair, rather than the increased induction of DNA damage, by the combinational treatment through RRM-1 activity could be responsible for the synergic effect of the combinational treatment with CDDP and 5-FU in TE11 cells.

\section{Discussion}

In the present study, we showed that RRM-1, large subunit of RNR involved in the metabolism of 5-FU to 5-FdU, is required for the induction of DNA damage by 5-FU. Interestingly, 5-FU treatment significantly increased the CDDP-induced RAD51 focus formation in an RRM-1-dependent manner. Taken together, these findings indicate that RRM-1 plays an important role in the anticancer effect of combinational therapy of 5-FU with CDDP through either the induction of DNA damage or modulation of DNA repair.

A significant synergistic inhibition of cell growth was observed after the combinational treatment with CDDP and 5-FU. A recent report showed that the retention of DNA damage-induced $\gamma$-H2AX foci and RAD51 foci is apparently 
A

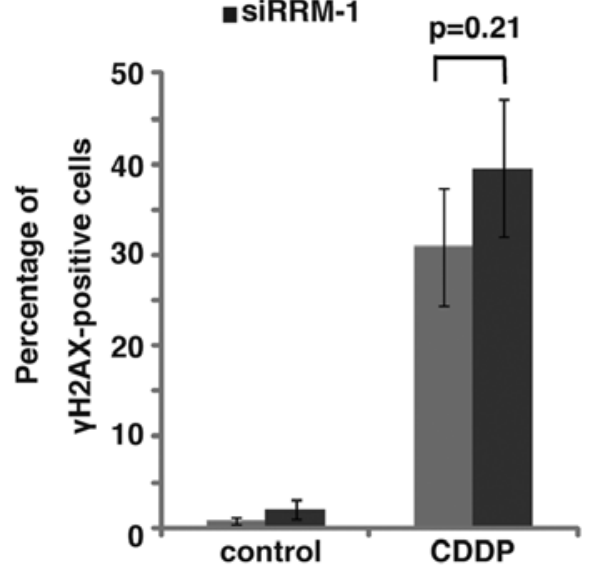

B

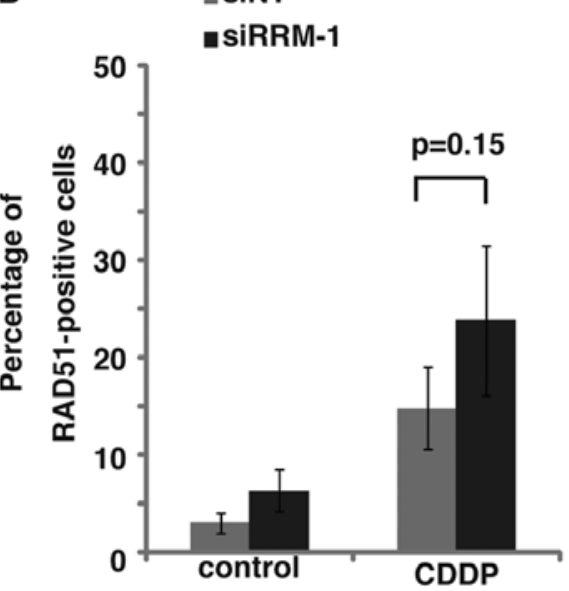

C
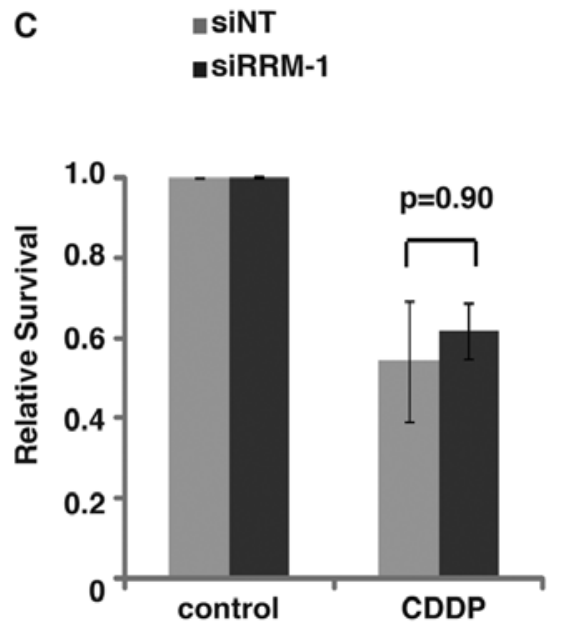

Figure 7. Effect of RRM-1 depletion on the induction of $\gamma$-H2AX and RAD51 focus formation in TE11 cells treated with CDDP alone. (A) Percentage of $\gamma$-H2AX foci-positive RRM-1 depleted cells (siRRM-1) and cells with treated with NTsiRNA (siNT), before and after CDDP treatment. All results are the means of three independent experiments, and error bars show the standard deviation of the mean. (B) Percentage of RAD51 foci-positive cells. All results are the means of three independent experiments, and error bars show the standard deviation of the mean. (C) The cytotoxicity of cells expressing either the NT siRNA or RRM-1 siRNA was determined, using the clonogenic assay. Results are the means of at least three independent experiments, and error bars show the standard deviation of the mean.

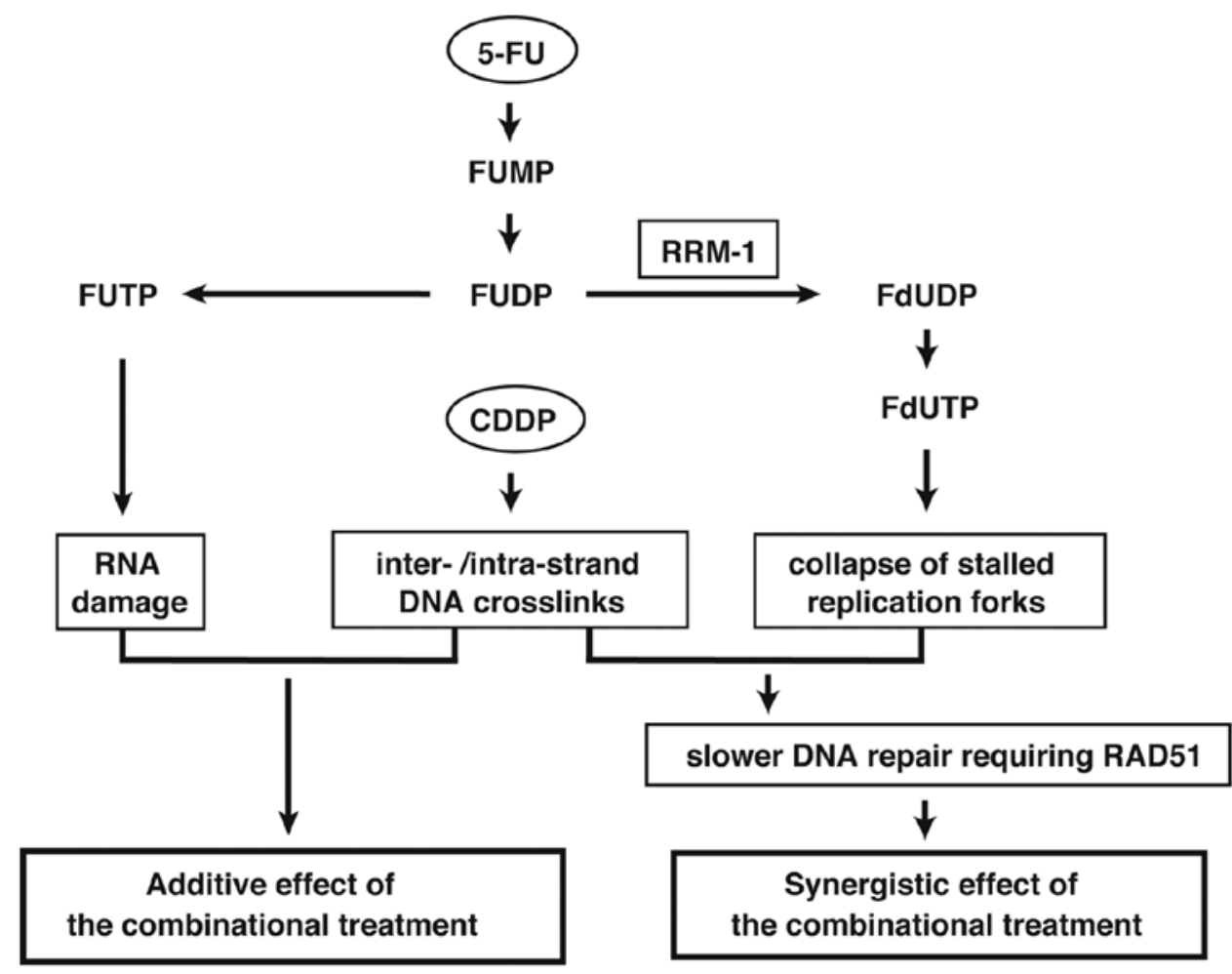

Figure 8 . The suggested mechanism of the anti-cancer effect by the 5-FU/CDDP combinational treatment. RRM-1 could enhance the synergistic effect by increasing the complex forms of DNA damage.

indicative of lethal DNA damage (29). The percentage of $\gamma$-H2AX-positive cells treated with the combination regimen was slightly higher than that treated with CDDP or 5-FU alone. Since both CDDP and 5-FU can induce DNA damage, this could be due to either the increased induction of DNA damage or the slowed DNA repair by the combinational treatment. The decline in the percentage of $\gamma$-H2AX-positive cells occurred earlier in the cells treated with CDDP, as compared to those subjected to the combinational treatment (Fig. 5B). Moreover, in contrast to the slight increase in $\gamma-\mathrm{H} 2 \mathrm{AX}$ foci positive cells, the percentage of TE11 cells with RAD51 foci was significantly increased after the combinational treatment (Fig. 5C). Although we could not exclude the possible role of the increased induction of DNA damage, these findings strongly support the notion that slower DNA repair, due to the combinational treatment, plays a role in the synergic effect in TE11 cells treated with 5-FU and CDDP. 
We found that RRM-1 plays important roles in the synergic effect of the combinational treatment with 5-FU and CDDP (Fig. 6A). However, the formation of $\gamma-\mathrm{H} 2 \mathrm{AX}$ foci in cells treated with the combinational regimen was not affected by the depletion of RRM-1 (Fig. 6D). In contrast to the $\gamma$-H2AX focus formation, the depletion of RRM-1 significantly reduced the focus formation by RAD51, a recombinational repair protein, after the combinational treatment (Fig. 6E). Since RRM-1 is required for the production of FdUDP and FdUTP which could disturb DNA metabolism including repair, these findings also support the notion that the synergic effect of the combinational treatment is due to slower DNA repair requiring RAD51 (Fig. 8).

In spite of the requirement of RRM-1 for the induction of DNA damage, the expression levels of RRM-1 did not affect the survival of TE11 cells after 5-FU alone treatment (Fig. 3E). Metabolites of 5-FU can disturb either DNA or RNA metabolisms (3). RRM-1 depletion could lead to increased conversion of 5-FU into 5-FUTP which contributes to the anticancer effect of 5-FU at several levels in RNA metabolism (30). The misincorporation of FUTP into RNA may inhibit the processing of pre-rRNA into mature rRNA $(31,32)$. In addition, it may disrupt the post-transcriptional modifications of tRNAs $(33,34)$ and the assembly and activity of snRNA/protein complexes, thus inhibiting the splicing of pre-mRNAs $(35,36)$. Therefore, the decreased induction of DNA damage by the depletion of RRM-1 after 5-FU treatment could be compensated by the increased disturbance of RNA metabolism, through the increased level of 5-FUTP in TE11 cells. Still further investigation to clarify the disturbance of RNA metabolism by the RRM-1 depletion is required, RRM-1 may play a key role in the regulation of the anticancer effect of 5-FU alone by disturbing either DNA or RNA metabolism.

In this study, we found that RRM-1 is involved in the regulation of the anticancer effect of 5-FU, in single and combinational treatments with CDDP. Although further studies are required to clarify the role of RRM-1 in the synergic effect of the combinational therapy, the expression level of RRM-1 may be a useful predictive marker for the treatment of patients with the combination regimen of CDDP and 5-FU. Moreover, the modulation of RRM-1 expression could be a novel therapeutic strategy to enhance the effects of CDDP and 5-FU.

\section{Acknowledgements}

This study was supported by the Grants-in-Aid Program from the Ministry of Education, Culture, Sports, Science and Technology of Japan.

\section{References}

1. Kamangar F, Dores GM and Anderson WF: Patterns of cancer incidence, mortality, and prevalence across five continents: defining priorities to reduce cancer disparities in different geographic regions of the world. J Clin Oncol 24: 2137-2150, 2006 .

2. Enzinger PC, Mayer RJ: Esophageal cancer. N Engl J Med 349: 2241-2252, 2003.

3. Longley DB, Harkin DP and Johnston PG: 5-fluorouracil: mechanisms of action and clinical strategies. Nat Rev Cancer 3: 330-338, 2003

4. Matuo R, Sousa FG, Escargueil AE, Grivicich I, GarciaSantos D, Chies JA, Saffi J, Larsen AK and Henriques JA: 5-Fluorouracil and its active metabolite FdUMP cause DNA damage in human SW620 colon adenocarcinoma cell line. J Appl Toxicol 29: 308-316, 2009.
5. Matuo R, Sousa FG, Escargueil AE, Soares DG, Grivicich I, Saffi J, Larsen AK and Henriques JA: DNA repair pathways involved in repair of lesions induced by 5 -fluorouracil and its active metabolite FdUMP. Biochem Pharmacol 79: 147-153, 2010.

6. Wyatt MD and Wilson DM III: Participation of DNA repair in the response to 5-fluorouracil. Cell Mol Life Sci 66: 788-799, 2009.

7. El-Awady RA, Saleh EM and Dahm-Daphi J: Targeting DNA double-strand break repair: is it the right way for sensitizing cells to 5-fluorouracil? Anticancer Drugs 21: 277-287, 2010.

8. Diasio RB and Johnson MR: Dihydropyrimidine dehydrogenase: its role in 5-fluorouracil clinical toxicity and tumor resistance. Clin Cancer Res 5: 2672-2673, 1999.

9. Mizutani Y, Wada H, Fukushima M, Yoshida O, Nakanishi H, Li YN and Miki T: Prognostic significance of orotate phosphoribosyltransferase activity in bladder carcinoma. Cancer 100: 723-731, 2004

10. Salonga D, Danenberg KD, Johnson M, Metzger R, Groshen S, Tsao-Wei DD, Lenz HJ, Leichman CG, Leichman L, Diasio RB and Danenberg PV: Colorectal tumors responding to 5-fluorouracil have low gene expression levels of dihydropyrimidine dehydrogenase, thymidylate synthase, and thymidine phosphorylase. Clin Cancer Res 6: 1322-1327, 2000.

11. Kolberg M, Strand KR, Graff P and Andersson KK: Structure, function, and mechanism of ribonucleotide reductases. Biochim Biophys Acta 1699: 1-34, 2004.

12. Niida H, Katsuno Y, Sengoku M, Shimada M, Yukawa M, Ikura M, Ikura T, Kohno K, Shima H, Suzuki H, Tashiro S and Nakanishi M: Essential role of Tip60-dependent recruitment of ribonucleotide reductase at DNA damage sites in DNA repair during G1 phase. Genes Dev 24: 333-338, 2010.

13. Rogakou EP, Pilch DR, Orr AH, Ivanova VS and Bonner WM: DNA double-stranded breaks induce histone $\mathrm{H} 2 \mathrm{AX}$ phosphorylation on serine 139. J Biol Chem 273: 5858-5868, 1998.

14. Uziel T, Lerenthal Y, Moyal L, Andegeko Y, Mittelman L and Shiloh Y: Requirement of the MRN complex for ATM activation by DNA damage. EMBO J 22: 5612-5621, 2003.

15. Branzei D and Foiani M: Regulation of DNA repair throughout the cell cycle. Nat Rev Mol Cell Biol 9: 297-308, 2008.

16. Arnaudeau C, Lundin C and Helleday T: DNA double-strand breaks associated with replication forks are predominantly repaired by homologous recombination involving an exchange mechanism in mammalian cells. J Mol Biol 307: 1235-1245, 2001.

17. Pardo B, Gomez-Gonzalez B and Aguilera A: DNA repair in mammalian cells: DNA double-strand break repair: how to fix a broken relationship. Cell Mol Life Sci 66: 1039-1056, 2009.

18. Suwaki N, Klare K and Tarsounas M: RAD51 paralogs: roles in DNA damage signalling, recombinational repair and tumorigenesis. Semin Cell Dev Biol 22: 898-905, 2011.

19. Tashiro S, Walter J, Shinohara A, Kamada N and Cremer T: Rad51 accumulation at sites of DNA damage and in postreplicative chromatin. J Cell Biol 150: 283-291, 2000.

20. Bolderson E, Richard DJ, Zhou BB and Khanna KK: Recent advances in cancer therapy targeting proteins involved in DNA double-strand break repair. Clin Cancer Res 15: 6314-6320, 2009.

21. Clingen PH, Wu JY, Miller J, Mistry N, Chin F, Wynne P, Prise KM and Hartley JA: Histone H2AX phosphorylation as a molecular pharmacological marker for DNA interstrand crosslink cancer chemotherapy. Biochem Pharmacol 76: 19-27, 2008.

22. Asakawa H, Koizumi H, Koike A, Takahashi M, Wu W, Iwase H, Fukuda M and Ohta T: Prediction of breast cancer sensitivity to neoadjuvant chemotherapy based on status of DNA damage repair proteins. Breast Cancer Res 12: R17, 2010.

23. Fujinaka Y, Matsuoka K, Iimori M, Tuul M, Sakasai R, Yoshinaga K, Saeki H, Morita M, Kakeji Y, Gillespie DA, Yamamoto K, Takata M, Kitao H and Maehara Y: ATR-Chk1 signaling pathway and homologous recombinational repair protect cells from 5-fluorouracil cytotoxicity. DNA Repair (Amst) 11: 247-258, 2010.

24. Scharer OD: DNA interstrand crosslinks: natural and druginduced DNA adducts that induce unique cellular responses. Chembiochem 6: 27-32, 2005.

25. Limoli CL, Giedzinski E, Bonner WM and Cleaver JE: $\mathrm{UV}$-induced replication arrest in the xeroderma pigmentosum variant leads to DNA double-strand breaks, gamma-H2AX formation, and Mre11 relocalization. Proc Natl Acad Sci USA 99: 233-238, 2002. 
26. Ewald B, Sampath D and Plunkett W: H2AX phosphorylation marks gemcitabine-induced stalled replication forks and their collapse upon S-phase checkpoint abrogation. Mol Cancer Ther 6: 1239-1248, 2007

27. Johnston PG, Geoffrey F, Drake J, Voeller D, Grem JL and Allegra CJ: The cellular interaction of 5-fluorouracil and cisplatin in a human colon carcinoma cell line. Eur J Cancer 32A: 2148-2154, 1996.

28. Matsusaka S, Nagareda $\mathrm{T}$ and Yamasaki $\mathrm{H}$ : Does cisplatin (CDDP) function as a modulator of 5-fluorouracil (5-FU) antitumor action? A study based on a clinical trial. Cancer Chemother Pharmacol 55: 387-392, 2005.

29. Banath JP, Klokov D, MacPhail SH, Banuelos CA and Olive PL: Residual gammaH2AX foci as an indication of lethal DNA lesions. BMC Cancer 10: 4, 2010.

30. Huehls AM, Wagner JM, Huntoon CJ, Geng L, Erlichman C, Patel AG, Kaufmann SH and Karnitz LM: Poly (ADP-Ribose) polymerase inhibition synergizes with 5-fluorodeoxyuridine but not 5-fluorouracil in ovarian cancer cells. Cancer Res 71: 4944-4954, 2011.
31. Kanamaru R, Kakuta H, Sato T, Ishioka C and Wakui A: The inhibitory effects of 5-fluorouracil on the metabolism of preribosomal and ribosomal RNA in L-1210 cells in vitro. Cancer Chemother Pharmacol 17: 43-46, 1986.

32. Ghoshal K and Jacob ST: Specific inhibition of pre-ribosomal RNA processing in extracts from the lymphosarcoma cells treated with 5-fluorouracil. Cancer Res 54: 632-636, 1994.

33. Santi DV and Hardy LW: Catalytic mechanism and inhibition of tRNA (uracil-5-) methyltransferase: evidence for covalent catalysis. Biochemistry 26: 8599-8606, 1987.

34. Randerath K, Tseng WC, Harris JS and Lu LJ: Specific effects of 5-fluoropyrimidines and 5-azapyrimidines on modification of the 5 position of pyrimidines, in particular the synthesis of 5-methyluracil and 5-methylcytosine in nucleic acids. Recent Results Cancer Res 84: 283-297, 1983.

35. Patton JR: Ribonucleoprotein particle assembly and modification of U2 small nuclear RNA containing 5-fluorouridine. Biochemistry 32: 8939-8944, 1993.

36. Doong SL and Dolnick BJ: 5-Fluorouracil substitution alters pre-mRNA splicing in vitro. J Biol Chem 263: 4467-4473, 1988. 